\title{
Situación socioeconómica de las familias afrodescendientes de los trabajadores en barcos turísticos del barrio "Punta Fría”, Ciudad de Bluefields, Nicaragua, durante el período $2008^{[15]}$
}

Grizeida Green Lincon $^{[16]}$

Heidi Guillen Romero[17]

\section{Resumen}

La presente investigación sirve de referencia a la sociedad costeña de los diferentes barrios de la ciudad de Bluefields. El objetivo general es evaluar el impacto que el fenómeno migratorio causa en relación a la situación socioeconómica de las familias afrodescendientes de los trabajadores en barcos turísticos (embarcados) del barrio "Punta Fría" de la ciudad de Bluefields, Nicaragua, durante el período 2008.

La investigación se desarrolló con la participación de las familias afrodescendientes del barrio "Punta Fría" que cuentan con un miembro embarcado; los embarcados que se encontraban de vacaciones y las agencias proveedores de fuente de empleo en los barcos turísticos. El enfoque metodológico utilizado para el desarrollo de esta investigación fue de carácter interpretativo, participativo y corte transversal, en complementariedad con los métodos cuantitativos y cualitativos.

La mayoría de las familias consultadas consideran que han mejorado en un $80 \%$ sus condiciones económicas de vida. Luego de analizar los efectos que recaen al seno familiar, se muestra que también se ha desintegrado la estabilidad familiar, porque los miembros de dichas familias permanecen más tiempo fuera del país que cerca de sus familias.

Las agencias han jugado un papel importante en la contratación de muchas personas para trabajar en estos barcos turísticos, brindándole a la población costeña una alternativa de subsistencia para las familias.

Palabras claves: Bluefields, personas trabajadoras embarcadas, situación socioeconómica de familiares.

[15] Monografía realizada con el financiamiento del proyecto PATHWAY II “Support to Graduation Research Projects of Indigenous and Afro Desendant Students in URACCAN, de la Fundación FORD.

[16] Licenciada en Administración de Empresas con mención en Recursos Naturales.

[17] Licenciada Heidi Guillén Romero, tutora de la investigación.

68 | CIENCIA E INTERCULTURALIDAD, Edición No 3, Diciembre 2008 


\section{Introducción}

El propósito del presente artículo es informar en relación al trabajo en los barcos turísticos, su importancia y aporte socioeconómico familiar, se fundamenta en las entrevistas con los familiares de las personas trabajadoras en barcos turísticos, la población en general del barrio "Punta Fría" y los embarcados que se encuentran de vacaciones.

Los beneficiaros directos de esta investigación son las familias del barrio "Punta Fría", que tienen alguno de sus miembros trabajando en los barcos turísticos; ellos, son los primeros que sufren de esta situación donde las hijas e hijos se quedan sin la madre o el padre, o las hermanas o hermanos adultos tienen que separase de la familia para ayudarles económicamente. Los beneficiaros indirectos es la población del barrio "Punta Fría". El trabajo en los barcos turísticos es la oportunidad más accesible especialmente para la población de habla inglés /creole.

El estudio se desarrolló en la ciudad de Bluefields ubicada a $383 \mathrm{Km}$ de Managua (2 hora de navegación en panga por el río Escondido hasta ciudad Rama; $292 \mathrm{Km}$ hasta la capital de Managua). La población de Bluefields es multiétnica, multilingüe y multicultural, distribuyéndose en seis etnias: miskitus, mestizos, sumos, creoles, garífunas, ramas. Cada grupo étnico habla su propio lenguaje y mantiene su propia cultura (Diagnóstico Municipalidad de Bluefields, 2000). Su población es de 43,909 habitantes aproximadamente (INEC., 2005).

El barrio "Punta Fría" (Cotton Tree) se encuentra ubicado geográficamente en la parte Sur de la ciudad de Bluefields, cabecera de la Región Autónoma del Atlántico Sur (RAAS). Es uno de los vecindarios más antiguos y también uno de los cuatro barrios de afrodescendientes o criollos como comúnmente los conocemos en esta ciudad.

\section{Revisión de literatura}

Los afrodescendientes que son objeto de este estudio, según el profesor Hugo Sujo Wilson (2008) tienen sus propias costumbres y tradiciones al igual que las otras cinco etnias: "Se define como criollo al individuo negro que se mezcla con cualquier otra raza, su característica principal es que son de habla inglesa, por regla general son protestantes y en Bluefields pertenecen en su mayoría a la religión Morava o Anglicana entre otras expresiones espirituales. Los alimentos o platillos en la mayoría de casos son preparados con la leche del coco con el cual aliñan el arroz y frijoles, conocido en la Costa Atlántica como el gallo pinto, además del rondón con variedades de carne. Su 
vestimenta en años anteriores estaba influenciada por el estilo inglés; antes se vestían como los caballeros ingleses y la moda Norte Americana, en sus creencias está que pertenecen a algunas logias (lodge) dentro del cual tienen sus propias celebraciones. Creen y practican el "obia" a lo que los mestizos conocen como el "something" que lo utilizan para realizar todo tipo de trabajo contra sus enemigos, en sus velorios o "Set Up" como se conoce en Inglés se caracterizan por cantar himnos religiosos durante toda la noche en la casa del difunto, reparten comida y bebidas a todos los que acompañan a la familia" .

En los 90's por el cambio de gobierno en Nicaragua y el auge de las actividades turísticas a nivel mundial se incrementan los números de barcos y por lo tanto las necesidades del personal y de ir a buscar empleo, comenzaron a reclutar consecutivamente por cinco años a trabajadores de la Costa Atlántica de Nicaragua en donde el encargado era el Ing. Wade Hawkins, el cual se independiza del sindicato de Marinos Mercantes para formar su propia agencia con la compañía de la Royal Caribbean que actualmente cuenta con más de 20 barcos cruceros que viajan a todos los rincones del mundo. Los cargos que estos ofrecen a los que optan por ir a trabajar en los barcos turísticos son los siguientes: Cleaner, Bar Server, Mess attendant, telephonist, cabin Stuard, Pool attendant, waiter en muchos casos es fácil aplicar a estos puestos una vez que se halla realizado la prueba de Inglés y se halla obtenido un alto puntaje, porque entre más capacidad de entender y comunicarse con el Ingles Standard es más fácil tener cargos (Ing. Wade Hawkins, Febrero 2008).

Es importante señalar que el Ing. Wade Hawkins hace mención que en 15 años de su agencia, ha logrado ayudar a más de 4,200 personas a obtener empleo.

En el año 1993 por el crecimiento de las actividades turísticas a nivel mundial y por ser la Costa Atlántica de Nicaragua proveedora de mano de obra calificada y especialmente de las personas de habla inglesa, aumentaron las compañías en nuestro país como son NCL (Norwegian Cruise Line), Mediterránea, Royal Caribbean International y Celebrity Cruise Line.(Ing. Wade Hawkins, Febrero 2008)

Actualmente existen 2 agencias que representan a estas compañías extranjeras como es la Agencia SETA (SEAMAN EXPRESS TRAVEL AGENCY) dirigida por el Ing. Wade Hawkins que representan las compañías extranjeras de NCL (Norwegian Cruise Line), Mediterranean y la Agencia Blue Rabbit dirigida por el Sr. Mike Wilson quien representa las compañías extranjeras de la Royal Caribbean International y Celebrity Cruise Line. Es importante mencionar que hace unos años atrás todas estas compañías eran representadas por el Ing. Wade Hawkins, y en ese entonces el Sr. Mike Wilson 
formaba parte del equipo de trabajo pero entre el Sr. Hawkins y el Sr. Wilson surgen conflictos internos.

\section{A continuación se incorporan los principales conceptos vinculados a la investigación}

Emigración: Es la entrada a un país o región por parte de personas que nacieron o proceden de otro lugar. Representa una de las dos opciones o alternativas del término migración, que se aplica a los movimientos de personas de un lugar a otro y estos desplazamientos conllevan un cambio de residencia bien sea temporal o definitivo. Las dos opciones de los movimientos migratorios son: Emigración, que es la salida de personas de un país, región o lugar determinados para dirigirse a otro distinto e inmigración, que es la entrada en un país, región o lugar determinados procedentes de otras partes. De manera que una emigración lleva como contrapartida posterior una inmigración en el país o lugar de llegada. (Wikipedía, 2008).

Socioeconómico: Que interesa a la sociedad definida en términos económicos. (Larousse, 2007). El cual respalda la investigación debido a que se estaría enmarcando sobre la situación económica en que viven los afrodescendientes con familias embarcadas.

Medio y proceso: Los medios son elementos o factores que condiciona la vida de un ser y los métodos o sistemas adoptados para llegar a un determinado fin. (Larousse, 2007).

Causa y efecto: Causa es el conjunto de circunstancia que determinan la situación de una persona y el efecto es el resultado de una acción o cosa que resulta en otra. (Larousse, 2007).

Embarcados: Son las personas que trabajan en los barcos cruceros o turísticos. Es un término popular que usa la población costeña para referirse a las personas que trabajan en los barcos turísticos.

Afrodescendientes: Pueblos de origen africano que fueron traídos como esclavos durante la colonia para sustituir la mano de obra de los pueblos indígenas exterminados en Estados Unidos y otros países del continente. Históricamente han sido víctimas de racismo, discriminación racial y esclavitud, con la negación reiterada de sus derechos humanos, condiciones que se encuentran en la base de la marginación, pobreza y exclusión que expresan la profunda desigualdad social y económica en la que viven. (Internet página de google fuente Instituto interamericano de Derechos Humanos). 


\section{Materiales y métodos}

El enfoque metodológico para el desarrollo de esta investigación fue de carácter interpretativo, participativo y de corte transversal.

De acuerdo con los objetivos definidos en el diseño metodológico, se considera el uso de dos técnicas cuantitativas: la revisión documental y la encuesta (Hernández \& Et al, 2000 $)^{[18]}$.

Para la revisión documental se consideraron como fuente: Diagnóstico de la Municipalidad de Bluefields, RAAS; Informe de Desarrollo Humano 2005, Datos poblacionales por barrios y grupos de edades del Ministerio de Salud y otras bibliografías para la realización del marco referencial y/o conceptual.

Con la revisión bibliográfica se puede establecer que hasta la fecha no hay documentación o indicios de estudio sobre las personas que trabajan en los barcos turísticos, sólo sobre la emigración y otros temas similares, el cual se pretende retomar para enriquecer la parte del marco referencial y/o conceptual de la investigación.

Las encuestas tienen una muestra de 148 familias afrodescendientes con miembro embarcado, de un total de 236 familias afrodescendientes del barrio de "Punta Fría", ciudad de Bluefields.

La muestra está formada por estratos diferentes que constituyen categorías importantes para la investigación: familias con miembros embarcados, los trabajadores de los barcos y un porcentaje de la comunidad para conocer su percepción en cuanto a los embarcados del mismo barrio. La muestra de hogares fue de forma aleatoria y luego se procedió a encuestar a las familias seleccionadas en forma sistemática.

\section{Procedimiento y recolección de datos}

Diseño de la encuesta: Para que los encuestados pudieran responder con rapidez y facilidad, las preguntas de la encuesta se elaboraron con preguntas claras y cortas.

Codificación de los datos recolectados: Se codificaron las preguntas de respuestas abiertas y cerradas para que los datos puedan ser leídos en programas computarizados.

[18] Hernández Sampieri, Fernández Collado, Baptista Lucio (2000). Metodología de la Investigación. Contratación fija y horaria. 
Trabajo de campo: El trabajo de campo se realizó en 45 viviendas del barrio "Punta Fría" de la ciudad de Bluefields, Nicaragua.

Procesamientos y análisis estadístico de los datos: Se procesaron los datos recopilados por medio de las encuestas a través del programa computarizado denominado Microsoft office Excell, 2007. Para la elaboración de los borradores y el informe final de la investigación se utilizó el programa Microsoft office Word, 2007.

\section{Resultado y discusión}

La mayoría de los familiares, conocidos y vecinos del barrio "Punta Fría" tienen una opinión positiva de las personas trabajadoras en los barcos turísticos en relación a la mejora de la situación socioeconómica, y lo consideran importante para el desarrollo de su hogar sobre todo en aspectos materiales.

Desde los primeros tiempos cuando se inició el proceso de reclutar personas para trabajar en barcos turísticos, las personas que optaron desde los 80`s para este tipo de empleo siempre incurrieron en algún tipo de gasto: sea pequeño o grande a través de un préstamo, sea de un familiar o una persona ajena a ellas, ya que para obtener este tipo de empleo siempre se ha tenido que contar con un capital para reunir los papeles y el boleto de viaje para el extranjero.

Según la información recopilada por medio de las entrevistas, el consenso existente en relación a las agencias proveedoras es que los medios y procesos de selección constituyen un aspecto positivo para el solicitante a la hora de decidir en qué agencia aplicar y ser seleccionado por medio de un sistema transparente. Las personas que han experimentado este proceso indican que han tenido que seguir las orientaciones indicadas por la agencia para poder tener en orden sus papeles y que son iguales, aunque las cantidades para los gastos en que se incurre pueden diferir.

Además existe una opinión similar tanto de las familias con miembros embarcados, como con los trabajadores de barcos y barcos turísticos que fueron entrevistados.

Existe relación entre los resultados de las encuestas y las entrevistas tanto entre la población del barrio "Punta Fría" y los embarcados del mismo barrio, sobre los cambios o efectos de tener un familiar embarcado y optar a este tipo de empleo. Sobre las causas por las cuales decidieron trabajar en barcos turísticos, la gran mayoría de los embarcados entrevistados indicaron que fue para poder ofrecer a sus familiares una mejor calidad de vida en esta ciudad. 


\section{MIGRACIONES}

Los trabajadores de los barcos turísticos del barrio "Punta Fría", concuerdan que se sienten satisfechos con el trabajo que tienen, con el trato de sus jefes y compañeros de trabajo y verifican el hecho de que sí existe tiempo para distraerse y recrearse en su hora de descanso.

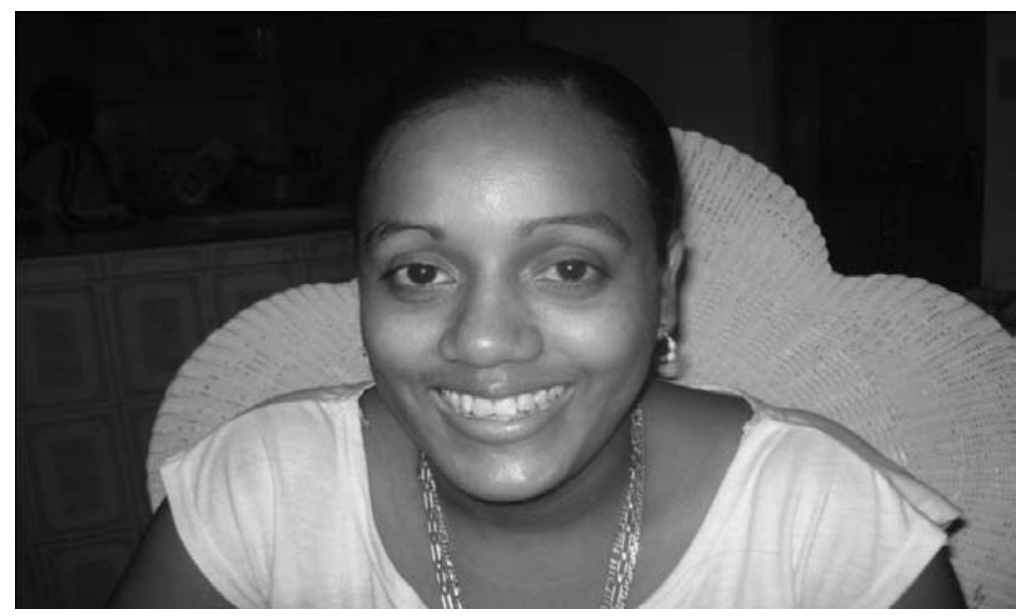

Figura 4: Sra. Suezannie Allen, esposa de un miembro embarcado del barrio "Punta Fría".

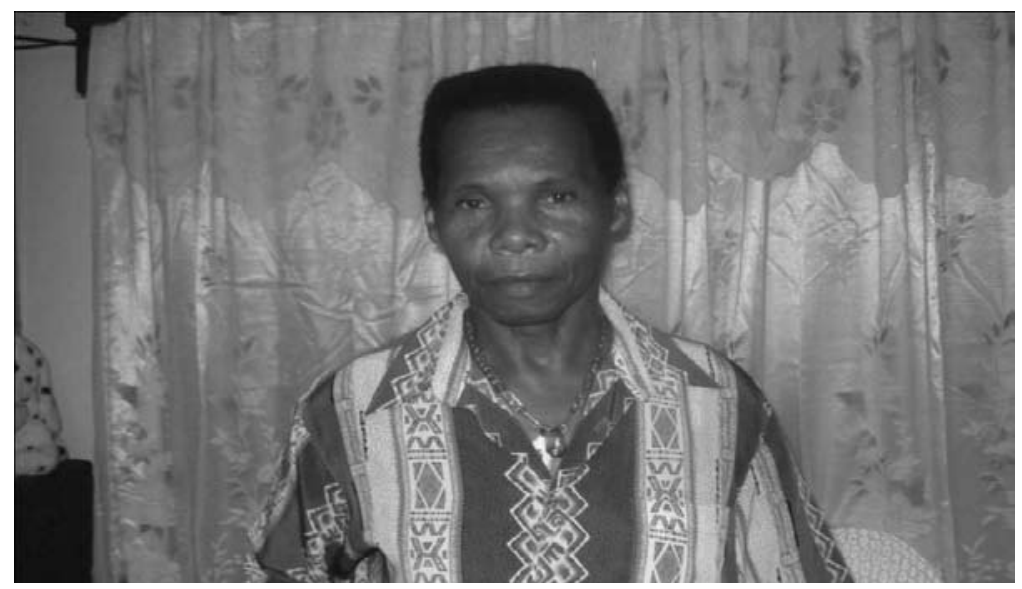

Figura 5: Sr. Frank Roe Garth, trabajador de barcos turísticos del barrio "Punta Fría" 


\section{Conclusiones}

La mayoría de las familias afrodescendientes con familiares embarcados describen su situación socioeconómica con mayor estabilidad de la que vivían anteriormente.

En tanto las agencias proveedoras que representan a los barcos turísticos dentro de la región, han desempeñado un papel muy importante apoyando a muchas familias afrodescendientes en la ciudad de Bluefields, al brindar por muchos años una alternativa de trabajo la cual consideran ellos mismos que no es la mejor opción; pero, por el alto índice de desempleo es necesario ya que es una forma de subsistencia de muchas familias. Aunque en los últimos dos años su foco de reclutamiento está siendo dirigido a la parte del Pacífico del país, por la experiencia que estos poseen en cuanto a trabajo de hoteles y restaurantes, lo cual está muy relacionada al trabajo en los barcos turísticos, también por entender y escribir el idioma Ingles Standard.

Este fenómeno que ha tenido mucha demanda en los últimos años, viene a cubrir muchos espacios de desempleo que se sufre en el país, causa principal por la cual muchas personas se van a trabajar en los barcos turísticos, para ofrecerle una mejor calidad de vida a sus familias y obtener los bienes materiales de una manera más rápida. Por otra parte ha causado efectos como la falta de permanencia de los estudiantes dentro de la Educación Secundaria e inclusive en superior como son las universidades, lo que vendrá a causar la falta de profesionales dentro de la región, aún sin tomar en cuenta que entre más preparados estén al para trabajar en un barco turístico, sus posibilidades de un mejor empleo aumentan.

Sin embargo, es importante mencionar que la situación laboral de los trabajadores en estos barcos turísticos muchas veces es difícil, porque son experiencias para las cuales en muchos casos los trabajadores no tienen un manejo concreto de los derechos humanos que ellos tienen como trabajadores. Aun así, muchas de las opiniones de los embarcados que fueron entrevistados están de acuerdo en que su situación ha mejorado desde que tienen estos empleos.

\section{Recomendaciones}

Los familiares afrodescendientes del barrio "Punta Fría" beneficiados del trabajo de sus miembros que están laborando en los barcos turísticos, que valoren aún más el esfuerzo que estos realizan, contribuyendo a minimizar gastos innecesarios y adquirir bienes materiales que realmente se necesitan y no que se desean. 
También que las personas que apliquen para ir a trabajar en los barcos turísticos tengan una mejor preparación en cuanto al manejo del idioma inglés, tanto en lo hablado como en lo escrito, teniendo así posibilidades de ser mejor promocionados en cuanto a cargos.

Las personas que están aplicando para embarcarse que puedan proponer a las agencias la posibilidad de obtener algún tipo de pasantías en los hoteles y restaurantes locales de buena calidad y servicios, para ir ganando experiencia relacionada al trabajo que se realizan en los barcos turísticos, de manera que nuestro país se mantenga como foco de reclutamiento de estas compañías extranjeras.

A las personas que trabajan en barcos turísticos tratar de invertir su dinero en alguna actividad que genere un ingreso fijo para ellos y sus familiares, a partir de pequeñas actividades que han logrado aprender al viajar por diferentes partes del mundo, con el objetivo de estabilizarse nuevamente con sus familiares.

A los miembros de familias afrodescendientes que trabajan en barcos turísticos motivar a sus hijos a obtener una educación superior completa, mientras ellos estén en la posibilidad de ofrecérsela.

A las agencias proveedoras que informen a las personas, que aplicaron y aprobaron para ir a trabajar en barcos turísticos, cuales son los derechos laborales que debe gozar un trabajador en estas compañías extranjeras.

\section{Lista de referencias}

Calero S. Valinda y Picon C. Astralia. (2000). Investigación es fácil II, manual de investigación. Edición Grisell Remegio. 2da edición. Universidad Nacional Autónoma de Nicaragua. UNAN- Managua: Editorial El amanecer.

Hernández Sampieri, Fernández Collado \& Baptista Lucio. (2000). Metodología de la Investigación. Mc Graw Hill.

Larousse (2007). Diccionario enciclopédico. Edición Larousse, S. A. 12 edición. México. 


\section{Lista de personas Entrevistadas}

Hawkins Wade (2008). Entrevista realizada por Grizeida Green, para recopilar información primaria.

Sujo Hugo. Profesor e historiador originario de Bluefields (2008). Entrevista realizada por la Investigadora Grizeida Green para recopilar información primaria.

Wilson Mike (2008) Entrevista realizada por investigadora Grizeida Green para recopilar información.

Google en internet (2008) consultado el 4 de Febrero 6:00 p.m.

Wikipedia (2007). Bluefields. Disponible en: http://es.wikipedia.org/wiki/Bluefields. Consultado el 2 de febrero a las 09:00 a.m. 